sciendo

\title{
KUMMER THEORY FOR NUMBER FIELDS AND THE REDUCTIONS OF ALGEBRAIC NUMBERS II
}

\author{
Antonella Perucca-Pietro Sgobba \\ University of Luxembourg, LUXEMBOURG
}

\begin{abstract}
Let $K$ be a number field, and let $G$ be a finitely generated and torsion-free subgroup of $K^{\times}$. For almost all primes $\mathfrak{p}$ of $K$, we consider the order of the cyclic group $(G \bmod \mathfrak{p})$, and ask whether this number lies in a given arithmetic progression. We prove that the density of primes for which the condition holds is, under some general assumptions, a computable rational number which is strictly positive. We have also discovered the following equidistribution property: if $\ell^{e}$ is a prime power and $a$ is a multiple of $\ell$ (and $a$ is a multiple of 4 if $\ell=2$ ), then the density of primes $\mathfrak{p}$ of $K$ such that the order of $(G \bmod \mathfrak{p})$ is congruent to $a$ modulo $\ell^{e}$ only depends on $a$ through its $\ell$-adic valuation.
\end{abstract}

\section{Communicated by Vladimír Baláž}

\section{Introduction}

If we reduce the number 2 modulo every odd prime number $p$, then we have the sequence of natural numbers given by the multiplicative order of $(2 \bmod p)$. This sequence is very mysterious, and for example it is not known unconditionally whether the order of $(2 \bmod p)$ equals $p-1$ for infinitely many primes $p$, see [3]. Now consider a non-zero integer $z$ : the density of primes $p$ for which the multiplicative order of $(z \bmod p)$ lies in a given arithmetic progression has been studied in various papers by Chinen and Murata, and by Moree, see, e.g. [1,4].

(C) 2020 BOKU-University of Natural Resources and Life Sciences and Mathematical Institute, Slovak Academy of Sciences.

2010 Mathematics Subject Classification: Primary: 11R44; Secondary: 11R45, 11R18, 11R21.

Keywords: Number field, reduction, multiplicative order, arithmetic progression, density. Licensed under the Creative Commons Attribution-NC-ND 4.0 International Public License. 


\section{ANTONELLA PERUCCA-PIETRO SGOBBA}

More generally, consider a number field $K$ and a multiplicative subgroup $G$ of $K^{\times}$which is finitely generated. For positive integers $x, y$ with $y \mid x$ we denote by $K_{x}:=K\left(\zeta_{x}\right)$ the $x$ th cyclotomic extension of $K$, and by $K_{x, y}:=K_{x}(\sqrt[y]{G})$ the $y$ th Kummer extension of $G$ over $K_{x}$. If $\mathfrak{p}$ is a prime of $K$, then we write $\operatorname{ord}_{\mathfrak{p}}(G)$ for the multiplicative order of $(G \bmod \mathfrak{p})$, which we tacitly assume to be well-defined. As customary, given two integers $x, y$ we write $(x, y)$ for their greatest common divisor and $[x, y]$ for their least common multiple. Finally, if we assume $(\mathrm{GRH})$ we mean the extended Riemann hypothesis for the Dedekind zeta function of number fields.

In [8] we have generalised results by Ziegler [10] to higher rank and have proven in particular the following statement.

Theorem 1 ([8, Theorem 1.3]). Let $K$ be a number field, and let $G$ be a finitely generated and torsion-free subgroup of $K^{\times}$of positive rank. Fix an integer $d \geqslant 2$, fix an integer $a$, and consider the following set of primes of $K$ :

$$
\mathcal{P}:=\left\{\mathfrak{p}: \operatorname{ord}_{\mathfrak{p}}(G) \equiv a \bmod d\right\} .
$$

Let $\mathcal{P}(x)$ be the number of primes $\mathfrak{p}$ in $\mathcal{P}$ with norm up to $x$.

Assuming (GRH), for every $x \geqslant 1$ we have

$$
\mathcal{P}(x)=\frac{x}{\log x} \sum_{n, t \geqslant 1} \frac{\mu(n) c(n, a, d, t)}{\left[K_{[d, n] t, n t}: K\right]}+O\left(\frac{x}{\log ^{3 / 2} x}\right),
$$

where $c(n, a, d, t) \in\{0,1\}$, and where $c(n, a, d, t)=1$ if and only if the following conditions hold:

(i) $(1+a t, d)=1$;

(ii) $(d, n) \mid a$;

(iii) the element of $\operatorname{Gal}\left(\mathbb{Q}\left(\zeta_{d t}\right) / \mathbb{Q}\right)$ mapping $\zeta_{d t}$ to $\zeta_{d t}^{1+a t}$ is the identity on $\mathbb{Q}\left(\zeta_{d t}\right) \cap$ $K_{n t, n t}$.

From this result it is not clear whether the natural density $\operatorname{dens}_{K}(G, a \bmod d)$ of the set $\mathcal{P}$ is a rational number, if it is strictly positive, or if it is possible to evaluate it. The main results of this paper are the following, where $K, G, a$, and $d$ are as in Theorem 1

TheOREM 2. Assume (GRH). Let $d=\ell^{e}$ for some prime number $\ell$ and for some $e \geqslant 1$. Suppose that $K=K_{\ell}$ if $\ell$ is odd, or that $K=K_{4}$ if $\ell=2$. Then the density $\operatorname{dens}_{K}\left(G, a\right.$ mod $\left.\ell^{e}\right)$ depends on a only through its $\ell$-adic valuation, and it is a computable strictly positive rational number. In particular, it is the same for all a coprime to $\ell$. 
Although the previous result has an assumption on the base field, we do not need that assumption in the following corollary.

Corollary 3 (Equidistribution property). Assume (GRH). Let $K$ be any number field, and let $d=\ell^{e}$ for a prime number $\ell$ and $e \geqslant 1$. Suppose that $\ell \mid$ a if $\ell$ is odd, or that $4 \mid a$ and $e \geqslant 2$ if $\ell=2$. Then the density $\operatorname{dens}_{K}\left(G, a \bmod \ell^{e}\right)$ depends on a only through its $\ell$-adic valuation, and it is a computable strictly positive rational number.

The following result concerns the case of composite modulus.

Theorem 4. Assume (GRH). Let $d \geqslant 2$ and set $r:=\prod_{\ell \mid d} \ell$ to be its radical. Suppose that $K=K_{r}$ if $d$ is odd, or that $K=K_{2 r}$ if $d$ is even. Then, for a coprime to $d$, the density $\operatorname{dens}_{K}(G, a \bmod d)$ is a computable strictly positive rational number which does not depend on a.

The following result generalises the positivity assertion of Corollary 3 .

Theorem 5. Assume (GRH). The density $\operatorname{dens}_{K}(G, a \bmod d)$ is strictly positive for any number field $K$ if $d$ is a prime power or if a is coprime to $d$.

Theorem 2 is proven in Section 3.1 for $\ell$ odd, and in Section 3.2 for $\ell=2$, respectively. We prove Corollary 3 in Section 3.3 . Theorem 4 is proven in Section [3.4, while Theorem [5] is proven in Section 3.5. Section 4 is devoted to removing from Theorem 1 the assumption that the group $G$ is torsion-free. Finally, Section 5 contains examples of applications of the above theorems and some numerical data.

Notice that in this paper we rely on Theorem 1 and hence most of our results assume (GRH): if the density in Theorem 1 is known unconditionally, then our results would also be unconditional.

\section{Preliminaries}

Let $K$ be a number field, and let $G$ be a finitely generated and torsion-free subgroup of $K^{\times}$. In the whole paper we tacitly assume that the primes $\mathfrak{p}$ of $K$ that we consider are such that the reduction of $G$ modulo $\mathfrak{p}$ is a well-defined subgroup of the multiplicative group of the residue field at $\mathfrak{p}$. Notice that the results of this section are unconditional. 


\section{ANTONELLA PERUCCA—PIETRO SGOBBA}

\subsection{Prescribing valuations for the order}

TheOREM 6. Let $\ell_{1}, \ldots, \ell_{n}$ be distinct prime numbers and $x_{1}, \ldots, x_{n}$ nonnegative integers. Then the density of primes $\mathfrak{p}$ of $K$ such that $v_{\ell_{i}}\left(\operatorname{ord}_{\mathfrak{p}}(G)\right)=x_{i}$ for all $i$ is a strictly positive computable rational number.

P r o of. The rationality of the density can be seen by neglecting the condition on the Frobenius in [5, Theorem 18]. For the positivity, apply [6, Proposition 12] to a basis $g_{1}, \ldots, g_{r}$ of $G$ consisting of $\mathbb{Z}$-independent points of the multiplicative group $K^{\times}$.

Corollary 7. Given an integer $d \geqslant 2$ and a positive divisor $g$ of $d$, the sum of densities

$$
\sum_{\substack{0 \leqslant a<d \\(a, d)=g}} \operatorname{dens}_{K}(G, a \bmod d)
$$

is a strictly positive computable rational number.

P r o o f. We will express the sum (2) as a rational combination of densities as in Theorem 6. Write $g=\prod_{i=1}^{n} \ell_{i}^{f_{i}}$, and partition the index set as $\{1, \ldots, n\}=I \sqcup J$ such that $f_{i}<v_{\ell_{i}}(d)$ for $i \in I$, and $f_{i}=v_{\ell_{i}}(d)$ for $i \in J$. Then it is easy to check that

$$
\sum_{\substack{0 \leqslant a<d \\
(a, d)=g}} \operatorname{dens}_{K}(G, a \bmod d)=\operatorname{dens}_{K}\left(\left\{\begin{array}{c}
v_{\ell_{i}}\left(\operatorname{ord}_{\mathfrak{p}}(G)\right)=f_{i}, \forall i \in I, \\
v_{\ell_{i}}\left(\operatorname{ord}_{\mathfrak{p}}(G)\right) \geqslant f_{i}, \forall i \in J
\end{array}\right\}\right) .
$$

From this expression and Theorem [6] we deduce that (2) is strictly positive. The density on the right-hand side of (3) is given by (applying the inclusion-exclusion principle for the primes up to $x$ and then taking the limit to make the densities)

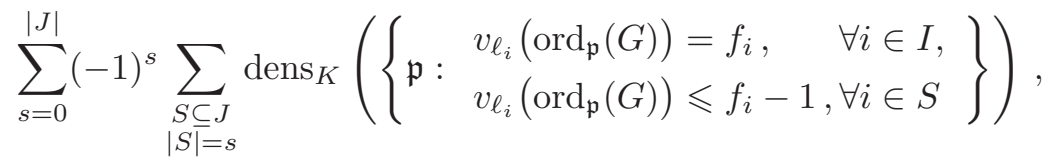

and each of the densities in (44) exists and equals

$$
\operatorname{dens}_{K}\left(\left\{\mathfrak{p}: \begin{array}{l}
v_{\ell_{i}}\left(\operatorname{ord}_{\mathfrak{p}}\left(G^{h}\right)\right)=f_{i}, \forall i \in I \\
\\
v_{\ell_{i}}\left(\operatorname{ord}_{\mathfrak{p}}\left(G^{h}\right)\right)=0, \forall i \in S
\end{array}\right\}\right)
$$

where $h=\prod_{i \in S} \ell_{i}^{f_{i}-1}$.

Such densities are computable rational numbers by Theorem 6 . Hence the statement is proven. 
REMARK 8. Corollary 7 implies that the density $\operatorname{dens}_{K}(G, 0 \bmod d)$ is known unconditionally to be a strictly positive computable rational number.

\subsection{Simplifications by changing the modulus}

We keep the notation of Theorem 1] By Remark 8 we may suppose that $0<a<d$. The following lemma allows us to reduce to residue classes coprime to $d$ if $d$ is a prime power.

Lemma 9. Let $d=\ell^{e}$, where $\ell$ is a prime number and $e \geqslant 1$. Suppose that $a=\ell^{x} \cdot w$, where $w$ is coprime to $\ell$ and $0<x<$ e. Set $w_{j}:=w+j \ell^{e-x}$ for $0 \leqslant j<\ell$ (notice that $w_{j}$ is also coprime to $\ell$ ). Then the primes $\mathfrak{p}$ of $K$ such that $\operatorname{ord}_{\mathfrak{p}}(G) \equiv a \bmod d$ are exactly those such that

minus those such that

$$
\operatorname{ord}_{\mathfrak{p}}\left(G^{\ell^{x}}\right) \equiv w \bmod \ell^{e-x}
$$

$$
\operatorname{ord}_{\mathfrak{p}}\left(G^{\ell^{x-1}}\right) \equiv w_{j} \bmod \ell^{e-x+1}
$$

for some $0 \leqslant j<\ell$. In particular, we have

$\operatorname{dens}_{K}\left(G, a \bmod \ell^{e}\right)=$

$$
\operatorname{dens}_{K}\left(G^{\ell^{x}}, w \bmod \ell^{e-x}\right)-\sum_{j=0}^{\ell-1} \operatorname{dens}_{K}\left(G^{\ell^{x-1}}, w_{j} \bmod \ell^{e-x+1}\right) .
$$

P r o of. Notice that condition (6) for any $j$ implies condition (5) because $w_{j}$ is coprime to $\ell$ and hence we must have $\operatorname{ord}_{\mathfrak{p}}\left(G^{\ell^{x}}\right)=\operatorname{ord}_{\mathfrak{p}}\left(G^{\ell^{x-1}}\right)$.

Let $\mathfrak{p}$ be a prime of $K$ such that $\operatorname{ord}_{\mathfrak{p}}(G) \equiv a \bmod d$. In particular, $\ell^{x}$ divides $\operatorname{ord}_{\mathfrak{p}}(G)$. Thus we have

$$
\operatorname{ord}_{\mathfrak{p}}\left(G^{\ell^{x}}\right)=\frac{\operatorname{ord}_{\mathfrak{p}}(G)}{\ell^{x}} \text { and } \operatorname{ord}_{\mathfrak{p}}\left(G^{\ell^{x-1}}\right)=\frac{\operatorname{ord}_{\mathfrak{p}}(G)}{\ell^{x-1}} .
$$

Dividing the congruence $\operatorname{ord}_{\mathfrak{p}}(G) \equiv a \bmod \ell^{e}$ by $\ell^{x}$ and $\ell^{x-1}$, respectively, we obtain

$$
\operatorname{ord}_{\mathfrak{p}}\left(G^{\ell^{x}}\right) \equiv w \bmod \ell^{e-x} \quad \text { and } \quad \operatorname{ord}_{\mathfrak{p}}\left(G^{\ell^{x-1}}\right) \equiv w \ell \bmod \ell^{e-x+1} .
$$

We have proven one containment because $w \ell$ is not congruent to any of the $w_{j}$ modulo $\ell$.

Now suppose that (5) holds, and that (6) does not hold for any $j$. In particular we must have $\operatorname{ord}_{\mathfrak{p}}\left(G^{\ell^{x-1}}\right) \neq \operatorname{ord}_{\mathfrak{p}}\left(G^{\ell^{x}}\right)$. We deduce $\operatorname{ord}_{\mathfrak{p}}\left(G^{\ell^{x-1}}\right)=\ell \cdot \operatorname{ord}_{\mathfrak{p}}\left(G^{\ell^{x}}\right)$, and therefore $\operatorname{ord}_{\mathfrak{p}}(G)=\ell^{x} \cdot \operatorname{ord}_{\mathfrak{p}}\left(G^{\ell^{x}}\right)$. We may conclude because multiplying (5) by $\ell^{x}$ gives

$$
\ell^{x} \cdot \operatorname{ord}_{\mathfrak{p}}\left(G^{\ell^{x}}\right) \equiv a \bmod d
$$




\section{ANTONELLA PERUCCA—PIETRO SGOBBA}

Remark 10. Consider the condition $\operatorname{ord}_{\mathfrak{p}}(G) \equiv a \bmod d$. Decompose $(a, d)=s h$ where $s=\prod_{\ell \mid(a, d)} \ell$ is its radical, and write $a^{\prime}=\frac{a}{h}, d^{\prime}=\frac{d}{h}$. Notice that $\left(a^{\prime}, d^{\prime}\right)=s$ is squarefree. We claim that the following equivalence holds:

$$
\operatorname{ord}_{\mathfrak{p}}(G) \equiv a \bmod d \quad \Longleftrightarrow \quad \operatorname{ord}_{\mathfrak{p}}\left(G^{h}\right) \equiv a^{\prime} \bmod d^{\prime} .
$$

If the first congruence is satisfied, then $(a, d)$ divides $\operatorname{ord}_{\mathfrak{p}}(G)$, so in particular we have

$$
\frac{\operatorname{ord}_{\mathfrak{p}}(G)}{h} \equiv a^{\prime} \bmod d^{\prime} \text {. }
$$

Since $h$ divides $\operatorname{ord}_{\mathfrak{p}}(G)$, we have $\frac{\operatorname{ord}_{\mathfrak{p}}(G)}{h}=\operatorname{ord}_{\mathfrak{p}}\left(G^{h}\right)$ and the second congruence holds. Conversely, if the second congruence is satisfied, then $s=\left(a^{\prime}, d^{\prime}\right)$ divides $\operatorname{ord}_{\mathfrak{p}}\left(G^{h}\right)$. Since $h$ introduces no new prime factors, we have

$$
\operatorname{ord}_{\mathfrak{p}}\left(G^{h}\right) \cdot h=\operatorname{ord}_{\mathfrak{p}}(G)
$$

and hence the congruence $\operatorname{ord}_{\mathfrak{p}}(G) \equiv a \bmod d$ holds.

\subsection{A general result}

We keep the notation from Theorem 1, and we denote by $\operatorname{Dens}_{K}(G, d)$ the density of primes $\mathfrak{p}$ of $K$ such that $\operatorname{ord}_{\mathfrak{p}}(G)$ is coprime to $d$.

REMarK 11. From the results in [2] and [7, under the assumptions of Theorems 2 and 4 the density $\operatorname{Dens}_{K}(G, d)$ depends on $G$ only through the $d$ -parameters for the $\ell$-divisibility of $G$ for each $\ell \mid d$. As a consequence of the results of this paper, the same holds for the density $\operatorname{dens}_{K}(G, a \bmod d)$ considered in Theorems 2 and 4 and in Corollary 3 ,

Theorem 12. Let $\ell$ be a prime number. Suppose that for every $G$ and for every $e \geqslant 1$ we have

$$
\operatorname{dens}_{K}\left(G, w \bmod \ell^{e}\right)=\operatorname{dens}_{K}\left(G, w^{\prime} \bmod \ell^{e}\right)
$$

as long as $w, w^{\prime}$ are coprime to $\ell$. Then for every $G$ and for every $e \geqslant 1$ the density

$$
\operatorname{dens}_{K}\left(G, a \bmod \ell^{e}\right)
$$

depends on a only through its $\ell$-adic valuation, and it is a computable rational number.

Pro of. We know from [2, Theorem 3] that the quantity

$$
\operatorname{Dens}_{K}(G, \ell)=1-\operatorname{dens}_{K}(G, 0 \bmod \ell)
$$

is a computable rational number. Then for every $a$ coprime to $\ell$, by the assumption on the equidistribution, we have

$$
\operatorname{dens}_{K}\left(G, a \bmod \ell^{e}\right)=\frac{1}{\varphi\left(\ell^{e}\right)} \cdot \operatorname{Dens}_{K}(G, \ell),
$$

so that $\operatorname{dens}_{K}\left(G, a \bmod \ell^{n}\right)$ is a computable rational number which does not depend on $a$. 


\section{KUMMER THEORY AND THE REDUCTIONS OF ALGEBRAIC NUMBERS II}

For $0<a<\ell^{e}$ not coprime to $\ell$ we apply Lemma 9, which allows us to compute the density $\operatorname{dens}_{K}\left(G, a \bmod \ell^{e}\right)$ as the difference of densities which we know to be computable rational numbers. More precisely, by the equidistribution condition the formula given in Lemma 9 becomes

$\operatorname{dens}_{K}\left(G, a \bmod \ell^{e}\right)=$

$$
\operatorname{dens}_{K}\left(G^{\ell^{x}}, w \bmod \ell^{e-x}\right)-\ell \cdot \operatorname{dens}_{K}\left(G^{\ell^{x-1}}, w \bmod \ell^{e-x+1}\right),
$$

where $a=w \ell^{x}$ and $x=v_{\ell}(a)$. In particular, this formula shows that what matters about $a$ is only its $\ell$-adic valuation.

Finally, the density for $a=0$ is given as the complementary density of all the considered cases, and hence it is also a computable rational number.

REMARK 13. Notice that for $0<a<\ell^{e}$ with some fixed valuation $v_{\ell}(a)=x$ where $0 \leqslant x<e$, the previous theorem says that we have the following density

$$
\operatorname{dens}_{K}\left(G, a \bmod \ell^{e}\right)=\frac{1}{\varphi\left(\ell^{e-x}\right)} \cdot \operatorname{dens}_{K}\left(\left\{\mathfrak{p}: v_{\ell}\left(\operatorname{ord}_{\mathfrak{p}}(G)\right)=x\right\}\right) .
$$

Proposition 14. With the assumptions of Theorem 12, we have that the density $\operatorname{dens}_{K}\left(G, a \bmod \ell^{e}\right)$ is strictly positive for every $a$.

Proof. For $a=0$ we know this unconditionally by Remark 8 . For $0<a<\ell^{e}$, by Theorem 6 the densities (17) in Remark 13 are strictly positive.

We say that a prime $\mathfrak{p}$ of $K$ is of degree 1 if both its ramification index and its residue class degree over $\mathbb{Q}$ are equal to 1 .

Lemma 15. Let $K$ be a number field, and let $G$ be a finitely generated and torsion-free subgroup of $K^{\times}$. Let $a, d$ be integers with $d \geqslant 2$ and let $r:=\prod_{\ell \mid d} \ell$ be the radical of $d$. Let $m=r$ if $d$ is odd, and $m=2 r$ otherwise. Consider the following set of primes $\mathfrak{p}$ of $K$ :

$$
\mathcal{S}:=\left\{\mathfrak{p}: \operatorname{ord}_{\mathfrak{p}}(G) \equiv a \bmod d, \mathrm{~N} \mathfrak{p} \equiv 1 \bmod m\right\} .
$$

Then the density of the set $\mathcal{S}$ exists and it is equal to

$$
\frac{1}{\left[K_{m}: K\right]} \cdot \operatorname{dens}_{K_{m}}(G, a \bmod d) \text {. }
$$

REMARK 16. Notice that, assuming $(\mathrm{GRH})$, a formula for the density of the set $\mathcal{S}$ is given in [8, Corollary 5.2]. By Theorems 2 and 4 it follows that the density (8) is a computable strictly positive rational number if $d$ is a prime power or if $a$ is coprime to $d$. Moreover, if $d=\ell^{e}$ for a prime $\ell$, then the density of $\mathcal{S}$ depends on $a$ only through its $\ell$-adic valuation, while if $d$ is composite and $(a, d)=1$, then it does not depend on $a$. 


\section{ANTONELLA PERUCCA-PIETRO SGOBBA}

Pr o of of L e m ma 15. We may assume that the primes $\mathfrak{p}$ of $\mathcal{S}$ are of degree 1 and unramified in $K_{m}$. Hence for a prime $\mathfrak{p}$ in $\mathcal{S}$ we have $\mathrm{Np} \equiv 1 \bmod m$ if and only if $\mathfrak{p}$ splits completely in $K_{m}$. Therefore, the set of primes of $K_{m}$ lying above the primes of $\mathcal{S}$ is the set

$$
\left\{\mathfrak{P} \subseteq K_{m} \text { of degree } 1: \operatorname{ord}_{\mathfrak{P}}(G) \equiv a \bmod d\right\}
$$

which has density $\operatorname{dens}_{K_{m}}(G, a \bmod d)$. Thus we obtain that the density of the set $\mathcal{S}$ exists and it is equal to $1 /\left[K_{m}: K\right]$ times $\operatorname{dens}_{K_{m}}(G, a \bmod d$ ) (see, for instance, [7, Proposition 1]).

\section{Proof of the results in the Introduction}

We keep the notation of Theorem 1

\subsection{Proof of Theorem 2 for $\ell$ odd}

Lemma 17. Let $\ell$ be an odd prime number. Suppose that $K=K_{\ell}$. For every $G$ and for every $e \geqslant 1$ we have

$$
c\left(n, x, \ell^{e}, t\right)=c\left(n, x^{\prime}, \ell^{e}, t\right)
$$

as long as $x, x^{\prime}$ are coprime to $\ell$.

P r o o f. Let $d=\ell^{e}$. Let $a$ vary among the integers strictly between 0 and $d$ and coprime to $\ell$. Since $a$ is coprime to $\ell$ and $d=\ell^{e}$, the condition $(d, n) \mid a$ means $\ell \nmid n$ and it is independent of $a$. If $c(n, a, d, t)$ is non-zero, then the integer $t$ must be divisible by $\ell$ because $\zeta_{\ell} \in K$ and hence it must be fixed if raised to the power $1+a t$ (recall that $a$ is coprime to $\ell$ ). In particular, the condition $(1+a t, d)=1$ holds independently of $a$.

We are left to check that Condition (iii) of Theorem 1 does not depend on $a$, provided that Conditions (i) and (ii) hold. Write $F:=K_{n t, n t}$ and define $\tau:=v_{\ell}(t)$. We thus have to show that the following is independent of $a$ : the Galois group of $F_{\ell^{e+\tau}} / F$ contains the automorphism $\sigma_{1+t a}$ satisfying $\zeta_{\ell^{e+\tau}} \mapsto \zeta_{\ell^{e+\tau}}^{1+a t}$. Since $K=K_{\ell}$, we have some largest integer $x \geqslant \tau \geqslant 1$ such that $F$ contains $\mathbb{Q}_{\ell^{x}}$, and this integer determines the Galois group of $F_{\ell^{e}+\tau} / F$, which is a finite cyclic $\ell$-group. 
If $x \geqslant e+\tau$, then the field extension $F_{\ell^{e+\tau}} / F$ is trivial and the coefficient $c\left(n, a, \ell^{e}, t\right)$ is 0 independently of $a$. Now suppose that $\tau \leqslant x<e+\tau$. The exponents for the action on $\zeta_{\ell^{e+\tau}}$ are those corresponding to the automorphisms of order dividing $\ell^{e+\tau-x}$. Since $v_{\ell}(a t)$ does not depend on $a$, we have that

$$
v_{\ell}\left((1+a t)^{\ell^{n}}-1\right)=\tau+n
$$

independently of $a$ and we conclude.

Proof of Theorem 2 for $\ell$ odd. Lemma 17implies that the conditions of Theorem 12 are satisfied if $K=K_{\ell}$ (compare with formula (1)). Thus the density $\operatorname{dens}_{K}(G, a \bmod d)$ depends on $a$ only through its $\ell$-adic valuation, and it is a computable rational number. By Proposition 14 this rational number must be strictly positive.

\subsection{Proof of Theorem 2 for $\ell=2$}

Lemma 18. Suppose $K=K_{4}$. For every $G$ and for every $e \geqslant 1$ we have

$$
c\left(n, x, 2^{e}, t\right)=c\left(n, x^{\prime}, 2^{e}, t\right)
$$

as long as $x, x^{\prime}$ are odd.

P r o of. Let $d=2^{e}$. Notice that the claim is clear for $e=1$, so suppose $e \geqslant 2$. Let $a$ vary in the odd integers strictly between 0 and $d$. Similarly to the proof of Lemma 17, the condition $(n, d) \mid a$ means that $2 \nmid n$ and is independent of $a$. Moreover, $t$ must be an even integer and hence the condition $(1+a t, d)=1$ is satisfied independently of $a$. Now suppose that the above conditions are satisfied, and let us focus on Condition (iii) of Theorem 1 .

Set $\tau:=v_{2}(t)$, and call $F$ the field $K_{n t, n t}$. Similarly to the proof of Lemma 17, we check that the following condition is independent of $a$ : the Galois group of $F_{2^{e+\tau}} / F$ contains the automorphism $\sigma_{1+t a}$ satisfying $\zeta_{2^{e+\tau}} \mapsto \zeta_{2^{e+\tau}}^{1+a t}$.

Recall that $K=K_{4}$, and call $x \geqslant 2$ the largest integer such that $F$ contains $\mathbb{Q}_{2^{x}}$ (we clearly have $x \geqslant \tau$ ). We then need to investigate the cyclic group $\operatorname{Gal}\left(\mathbb{Q}_{2^{e+\tau}} / \mathbb{Q}_{2^{x}}\right)$.

If $x \geqslant e+\tau$, then this field extension is trivial and we have $c\left(n, a, 2^{e}, t\right)=0$ independently of $a$ (where $a$ is odd). If $x=\tau$, then $\operatorname{Gal}\left(\mathbb{Q}_{2^{e+\tau}} / \mathbb{Q}_{2^{x}}\right)$ contains $2^{e}$ automorphisms acting distinctly on $\zeta_{2^{e+\tau}}$ and fixing $\zeta_{2^{\tau}}$ : we deduce that $c\left(n, a, 2^{e}, t\right)=1$ independently of $a$ (where $a$ is odd).

From now on, suppose $\tau<x<e+\tau$. We see $\operatorname{Gal}\left(\mathbb{Q}_{2^{e+\tau}} / \mathbb{Q}_{2^{x}}\right)$ as a subgroup of the cyclic Galois group $\operatorname{Gal}\left(\mathbb{Q}_{2^{e+\tau}} / \mathbb{Q}_{4}\right)$. That subgroup contains the elements of order dividing $2^{e+\tau-x}$. The Galois automorphisms are determined by the image of $\zeta_{2^{e+\tau}}$, and they are determined by the exponent to which they raise this element. 
If $\tau=1$, then we do not have the automorphism $\sigma_{1+t a}$ in $\operatorname{Gal}\left(\mathbb{Q}_{2^{e+\tau}} / \mathbb{Q}_{4}\right)$ (independently of $a$ ) because $a$ is odd and hence $\zeta_{4}^{1+t a} \neq \zeta_{4}$. This means that in this case $c\left(n, a, 2^{e}, t\right)=0$ independently of $a$ (for $a$ odd).

Finally, suppose $1<\tau<x<e+\tau$. Since $\tau>1$, the automorphism $\sigma_{1+t a} \in$ $\operatorname{Gal}\left(\mathbb{Q}_{2^{e+\tau}} / \mathbb{Q}_{4}\right)$ is well-defined. We have to check whether $\sigma_{1+t a}$ also belongs to $\operatorname{Gal}\left(\mathbb{Q}_{2^{e+\tau}} / \mathbb{Q}_{2^{x}}\right)$ or not independently of $a$. It is then sufficient to show that the order of $\sigma_{1+t a}$ does not depend on $a$. This order is a power of 2 , namely the smallest power $2^{n}$ such that $v\left((1+a t)^{2^{n}}-1\right) \geqslant e+\tau$. Since $v_{2}(a t) \geqslant 2$, then for every $n \geqslant 1$ we have $v_{2}\left((1+a t)^{2^{n}}-1\right)=\tau+n$ independently of $a$ and hence the order of the automorphism $\sigma_{1+t a}$ does not depend on $a$.

Pro of of Theorem 2 for $\ell=2$. Analogously to the proof for the odd case, it suffices to combine Lemma 18 with Theorem 12 and Proposition 14.

\subsection{Proof of Corollary 3}

Pro of of Corollary [3, Let $m=\ell$ if $\ell$ is odd, and $m=4$ if $\ell=2$. Let $\mathfrak{p}$ be a prime of $K$ of degree 1 , and which does not ramify in $K_{m}$. In view of our hypothesis on $a$, we have that if $\mathfrak{p}$ is such that $\operatorname{ord}_{\mathfrak{p}}(G) \equiv a \bmod \ell^{e}$, then $\mathrm{Np} \equiv 1 \bmod m$. We deduce from Lemma 15, that

$$
\operatorname{dens}_{K}\left(G, a \bmod \ell^{e}\right)=\frac{1}{\left[K_{m}: K\right]} \cdot \operatorname{dens}_{K_{m}}\left(G, a \bmod \ell^{e}\right) .
$$

By Theorem 2 we conclude that $\operatorname{dens}_{K}\left(G, a \bmod \ell^{e}\right)$ depends on $a$ only through its $\ell$-adic valuation and that it is a computable strictly positive rational number.

\subsection{Proof of Theorem 4}

Lemma 19. Let $d \geqslant 2$ be an integer and write $d=\prod \ell^{e}$ for its prime decomposition. For the coefficients of Theorem 1, with respect to any fixed group $G$, we have

$$
c(n, a, d, t)=\prod_{\ell \mid d} c\left(n, a, \ell^{e}, t\right) .
$$

Proof. We prove that $c(n, a, d, t)=1$ if and only if $c\left(n, a, \ell^{e}, t\right)=1$ for every prime divisor $\ell$ of $d$. It is clear that $(1+a t, d)=1$ and $(d, n) \mid a$ if and only if $\left(1+a t, \ell^{e}\right)=1$ and $\left(\ell^{e}, n\right) \mid a$ for every $\ell$. Now suppose that these conditions hold. Let $\sigma$ be the element of $\operatorname{Gal}\left(\mathbb{Q}\left(\zeta_{d t}\right) / \mathbb{Q}\right)$ such that $\sigma\left(\zeta_{d t}\right)=\zeta_{d t}^{1+a t}$, and let $\sigma_{\ell}$ be the element of $\operatorname{Gal}\left(\mathbb{Q}\left(\zeta_{\ell^{e} t}\right) / \mathbb{Q}\right)$ such that $\sigma_{\ell}\left(\zeta_{\ell^{e} t}\right)=\zeta_{\ell^{e} t}^{1+a t}$. We are left to show that $\sigma$ is the identity on $\mathbb{Q}\left(\zeta_{d t}\right) \cap K_{n t, n t}$ if and only if $\sigma_{\ell}$ is the identity on $\mathbb{Q}\left(\zeta_{\ell^{e} t}\right) \cap K_{n t, n t}$ for every $\ell$. This follows from the fact that $\mathbb{Q}\left(\zeta_{d t}\right)$ is the compositum of the fields $\mathbb{Q}\left(\zeta_{\ell^{e} t}\right)$, and $\sigma_{\ell}$ is the restriction of $\sigma$ to $\mathbb{Q}\left(\zeta_{\ell^{e}}\right)$ for each $\ell$. 
LEMma 20. Let $d \geqslant 2$ be an integer and let $r:=\prod_{\ell \mid d} \ell$ be its radical. Suppose that $K=K_{r}$ if $d$ is odd, or that $K=K_{2 r}$ if $d$ is even. For the coefficients of Theorem 1, with respect to any fixed group $G$, we then have

$$
c(n, x, d, t)=c\left(n, x^{\prime}, d, t\right)
$$

as long as $x, x^{\prime}$ are coprime to $d$.

Proof. We have to show that, whenever $a$ is coprime to $d$, the coefficient $c(n, a, d, t)$ is independent of $a$. By Lemma 19 we may reduce to the case in which $d$ is a prime power, and then we may conclude by Lemma 17 if $d$ is odd, and Lemma 18 if $d$ is even.

Proof of Theorem 4, By [7, Corollary 12] and [2, Theorem 3] the density $\operatorname{Dens}_{K}(G, d)$ of primes $\mathfrak{p}$ of $K$ such that $\operatorname{ord}_{\mathfrak{p}}(G)$ is coprime to $d$ is an explicitly computable rational number. This density can be decomposed as the sum over $a$, with $a$ coprime to $d$, of the densities $\operatorname{dens}_{K}(G, a \bmod d)$. Since $K_{r}=K$ if $d$ is odd, and $K_{2 r}=K$ if $d$ is even, by Lemma 20 the above densities have equal value, so that for every a coprime to $d$ we have

$$
\operatorname{dens}_{K}(G, a \bmod d)=\frac{1}{\varphi(d)} \cdot \operatorname{Dens}_{K}(G, d),
$$

which is then a computable rational number. Moreover, this density is also strictly positive because by Theorem 6 the density $\operatorname{Dens}_{K}(G, d)$ is strictly positive.

\subsection{Proof of Theorem 5}

Proof of Theorem [5. Let $r$ be the radical of $d$, and let $m=r$ if $d$ is odd, and $m=2 r$ otherwise. Consider the following set of primes $\mathfrak{p}$ of $K$ of degree 1 , and unramified in $K_{m}$

$$
\mathcal{S}:=\left\{\mathfrak{p}: \operatorname{ord}_{\mathfrak{p}}(G) \equiv a \bmod d, \mathrm{~Np} \equiv 1 \bmod m\right\} .
$$

By Lemma 15 the set $\mathcal{S}$ has density equal to

$$
\frac{1}{\left[K_{m}: K\right]} \cdot \operatorname{dens}_{K_{m}}(G, a \bmod d) .
$$

By Theorems 2 and 4, the density $\operatorname{dens}_{K_{m}}(G, a \bmod d)$ is strictly positive if $d$ is a prime power or if $a$ is coprime to $d$, so the same holds for the density of $\mathcal{S}$. Consequently, the density $\operatorname{dens}_{K}(G, a \bmod d)$ is also strictly positive. 


\section{Multiplicative groups with torsion}

Stating Theorem 1 for a finite group is trivial (the given density is either 0 or 1). However it is not trivial to remove the assumption that the multiplicative group is torsion-free: this is what we achieve in this section. As a side remark, notice that our strategy also applies to the density considered in [8, Theorem 1.4], i.e. if we introduce a condition on the Frobenius conjugacy class with respect to a fixed finite Galois extension of the base field.

Let $K$ be a number field, and let $G^{\prime}$ be a finitely generated (and not necessarily torsion-free) multiplicative subgroup of $K^{\times}$of positive rank. Then we can write $G^{\prime}$ as $G^{\prime}=\langle\zeta\rangle \times G$, where $\zeta$ is a root of unity of $K$ generating the torsion part of $G^{\prime}$ and $G$ is torsion-free. Let us exclude finitely many primes $\mathfrak{p}$ of $K$ so that the reduction of $G^{\prime}$ is well-defined and we have $\operatorname{ord}_{\mathfrak{p}}(\zeta)=\operatorname{ord}(\zeta)$. The order of $G^{\prime}$ modulo $\mathfrak{p}$ is then the least common multiple between the order of $G$ modulo $\mathfrak{p}$ and a fixed integer

$$
\operatorname{ord}_{\mathfrak{p}}\left(G^{\prime}\right)=\left[\operatorname{ord}_{\mathfrak{p}}(G), \operatorname{ord}(\zeta)\right]
$$

We may then reformulate the given problem.

REMARK 21. Let $G$ be a finitely generated and torsion-free subgroup of $K^{\times}$, and fix some integer $n \geqslant 2$. Given two integers $a$ and $d \geqslant 2$, we investigate the density of primes $\mathfrak{p}$ of $K$ for which

$$
\left[\operatorname{ord}_{\mathfrak{p}}(G), n\right] \equiv a \bmod d .
$$

Assuming (GRH), the case $n=1$ is known, and our aim is reducing to this case. Notice that our method also shows that the considered density exists. We denote this density by $\operatorname{dens}_{K}^{\prime}(G, n ; a \bmod d)$.

Let $\ell$ be a prime divisor of $n$. The aim is finding a way to replace $n$ with $\frac{n}{\ell}$ (or to conclude directly). We distinguish various cases.

CAse (i). If $\ell \mid d$ and $\ell \nmid a$, then we have $\operatorname{dens}_{K}^{\prime}(G, n ; a \bmod d)=0$ because $\ell$ divides $\left[\operatorname{ord}_{\mathfrak{p}}(G), n\right]$ and (9) cannot hold.

CAse (ii). If $\ell \mid d$ and $\ell \mid a$, then the congruence $\left[\operatorname{ord}_{\mathfrak{p}}(G), n\right] \equiv a \bmod d$ is equivalent to

so we have

$$
\left[\operatorname{ord}_{\mathfrak{p}}\left(G^{\ell}\right), \frac{n}{\ell}\right] \equiv \frac{a}{\ell} \bmod \frac{d}{\ell},
$$

$$
\operatorname{dens}_{K}^{\prime}(G, n ; a \bmod d)=\operatorname{dens}_{K}^{\prime}\left(G^{\ell}, \frac{n}{\ell} ; \frac{a}{\ell} \bmod \frac{d}{\ell}\right) .
$$




\section{KUMMER THEORY AND THE REDUCTIONS OF ALGEBRAIC NUMBERS II}

CASE (iii). Suppose that $\ell \nmid d$. Let $\tilde{\ell}$ be a multiplicative inverse for $\ell$ modulo $d$, and set $v:=v_{\ell}(n)$. If $\ell^{v} \mid \operatorname{ord}_{\mathfrak{p}}(G)$, then we have

$$
\left[\operatorname{ord}_{\mathfrak{p}}(G), n\right] \equiv a \bmod d \quad\left[\operatorname{ord}_{\mathfrak{p}}(G), \frac{n}{\ell}\right] \equiv a \bmod d .
$$

If $\ell^{v} \nmid \operatorname{ord}_{\mathfrak{p}}(G)$, then we have

$$
\left[\operatorname{ord}_{\mathfrak{p}}(G), n\right] \equiv a \bmod d \quad \Longleftrightarrow \quad\left[\operatorname{ord}_{\mathfrak{p}}(G), \frac{n}{\ell}\right] \equiv a \tilde{\ell} \bmod d .
$$

The condition $\ell^{v} \mid \operatorname{ord}_{\mathfrak{p}}(G)$ amounts to

$$
\left[\operatorname{ord}_{\mathfrak{p}}(G), \frac{n}{\ell}\right] \equiv 0 \bmod \ell^{v}
$$

and hence (recalling that $\ell$ and $d$ are coprime) we can easily combine this congruence and the congruence in (10) with the Chinese Remainder Theorem. The first subcase thus amounts to

$$
\left[\operatorname{ord}_{\mathfrak{p}}(G), \frac{n}{\ell}\right] \equiv a \tilde{\ell}^{v} \ell^{v} \bmod d \ell^{v}
$$

Similarly, the second subcase amounts to letting $\left[\operatorname{ord}_{\mathfrak{p}}(G), \frac{n}{\ell}\right]$ be in the difference of congruence classes

$$
(a \tilde{\ell} \bmod d) \backslash\left(a \tilde{\ell}^{v+1} \ell^{v} \bmod d \ell^{v}\right) .
$$

Notice that the congruence classes for the first and second subcase are distinct. Thus if $\ell \nmid d$ we can explicitly write

$$
\begin{aligned}
\operatorname{dens}_{K}^{\prime}(G, n ; a \bmod d)= & \operatorname{dens}_{K}^{\prime}\left(G, \frac{n}{\ell} ; a_{0} \bmod d \ell^{v}\right) \\
& +\operatorname{dens}_{K}^{\prime}\left(G, \frac{n}{\ell} ; a \tilde{\ell} \bmod d\right) \\
& -\operatorname{dens}_{K}^{\prime}\left(G, \frac{n}{\ell} ; a_{0} \tilde{\ell} \bmod d \ell^{v}\right),
\end{aligned}
$$

where we have set $a_{0}:=a \tilde{\ell}^{v} \ell^{v} \bmod d \ell^{v}$.

We have thus proven the following result.

Theorem 22. Assume (GRH). Let $K$ be a number field, and let $G^{\prime}$ be a finitely generated subgroup of $K^{\times}$of positive rank. Let $n \geqslant 1$ be the order of the torsion of $G^{\prime}$, and let $G$ be a torsion-free subgroup of $G^{\prime}$ such that $G^{\prime}=G \times\left\langle\zeta_{n}\right\rangle$. Let $a$ and $d \geqslant 2$ be fixed integers. The density of the set of primes $\mathfrak{p}$ of $K$

$$
\left\{\mathfrak{p}: \operatorname{ord}_{\mathfrak{p}}\left(G^{\prime}\right) \equiv a \bmod d\right\}
$$

exists and can be expressed as a finite sum of terms of the type

$$
(-1)^{k} \cdot \operatorname{dens}_{K}\left(G^{m}, a^{\prime} \bmod d^{\prime}\right),
$$

where $k, m, a^{\prime}, d^{\prime}$ are integers and $m \mid n$. 


\section{ANTONELLA PERUCCA-PIETRO SGOBBA}

\section{Examples}

In this last section we work out some examples and collect some numerical data to illustrate our results.

EXAmple 23. Let $K=\mathbb{Q}\left(\zeta_{3}\right)$ and consider the group $G=\langle 5,7\rangle \leqslant \mathbb{Q}\left(\zeta_{3}\right)^{\times}$. We compute the density

$$
\operatorname{dens}_{K}(G, a \bmod 9) \text { for } 0 \leqslant a<9 .
$$

Since $\zeta_{3} \in K$, we can use [2, Theorem 2] to compute the density of primes $\mathfrak{p}$ of $K$ for which the order of $G \bmod \mathfrak{p}$ is coprime to 3 , and we have

$$
\operatorname{Dens}_{K}(G, 3)=\frac{1}{13} \text {. }
$$

Then by Theorem 2 we have

$$
\operatorname{dens}_{K}(G, a \bmod 9)=\frac{1}{78} \text { for } a \in\{1,2,4,5,7,8\} .
$$

For $a=3$ or $a=6$, by [2, Theorem 3] we have

$$
\operatorname{Dens}_{K}\left(G^{3}, 3\right)=\frac{9}{13}
$$

and applying Lemma 9 we obtain by the equidistribution property

$$
\begin{aligned}
\operatorname{dens}_{K}(G, a \bmod 9) & =\operatorname{dens}_{K}\left(G^{3}, 1 \bmod 3\right)-3 \operatorname{dens}_{K}(G, 1 \bmod 9) \\
& =\frac{9}{2 \cdot 13}-3 \cdot \frac{1}{78}=\frac{4}{13} .
\end{aligned}
$$

For $a=0$ we get the complementary density of $\operatorname{Dens}_{K}\left(G^{3}, 3\right)$ and hence

$$
\operatorname{dens}_{K}(G, 0 \bmod 9)=\frac{4}{13} .
$$

EXAMPLE 24. Let $K=\mathbb{Q}\left(\zeta_{4}\right)$ and consider the group $G=\langle 3,5\rangle \leqslant \mathbb{Q}\left(\zeta_{4}\right)^{\times}$. We compute the density of primes $\operatorname{dens}_{K}(G, a \bmod 8)$ for $0 \leqslant a<8$. Since $\zeta_{4} \in K$, by [2, Theorem 2] the density of primes $\mathfrak{p}$ of $K$ for which the order of $G \bmod \mathfrak{p}$ is odd is given by

$$
\operatorname{Dens}_{K}(G, 2)=\frac{1}{28} \text {. }
$$

Then by Theorem 2 we have

$$
\operatorname{dens}_{K}(G, a \bmod 8)=\frac{1}{112} \text { for } a \in\{1,3,5,7\} .
$$




\section{KUMMER THEORY AND THE REDUCTIONS OF ALGEBRAIC NUMBERS II}

For $a=2$ or $a=6$, by [2, Theorem 3] we have

$$
\operatorname{Dens}_{K}\left(G^{2}, 2\right)=\frac{1}{7},
$$

and applying Lemma 9 we obtain by the equidistribution property

$$
\begin{aligned}
\operatorname{dens}_{K}(G, a \bmod 8) & =\operatorname{dens}_{K}\left(G^{2}, 1 \bmod 4\right)-2 \operatorname{dens}_{K}(G, 1 \bmod 8) \\
& =\frac{1}{14}-2 \cdot \frac{1}{112}=\frac{3}{56} .
\end{aligned}
$$

For $a=4$ we proceed similarly. By [2, Theorem 3] we have

$$
\operatorname{Dens}_{K}\left(G^{4}, 2\right)=\frac{4}{7}
$$

and then, by Lemma 9, we obtain by the equidistribution property

$$
\begin{aligned}
\operatorname{dens}_{K}(G, 4 \bmod 8) & =\operatorname{dens}_{K}\left(G^{4}, 1 \bmod 2\right)-2 \operatorname{dens}_{K}\left(G^{2}, 1 \bmod 4\right) \\
& =\frac{4}{7}-\frac{1}{7}=\frac{3}{7} .
\end{aligned}
$$

Finally, for $a=0$ we obtain the complementary density

$$
\operatorname{dens}_{K}(G, 0 \bmod 8)=\frac{3}{7} .
$$

EXAMPLE 25. Let $K=\mathbb{Q}\left(\zeta_{12}\right)$ and consider the group $G=\langle 7,11\rangle \leqslant \mathbb{Q}\left(\zeta_{12}\right)^{\times}$. We compute the density of primes $\operatorname{dens}_{K}(G, a \bmod 12)$ for $a \in\{1,5,7,11\}$, which are all equal by Theorem 4 as $\zeta_{12} \in K$. By [7, Corollary 12] the density of primes $\mathfrak{p}$ of $K$ for which the order of $G \bmod \mathfrak{p}$ is coprime to 12 can be computed as in the previous examples

$$
\operatorname{Dens}_{K}(G, 12)=\operatorname{Dens}_{K}(G, 4) \cdot \operatorname{Dens}_{K}(G, 3)=\frac{1}{364} .
$$

Hence we obtain by the equidistribution

$$
\operatorname{dens}_{K}(G, a \bmod 12)=\frac{1}{1456} .
$$

In the following two examples we also compute with SageMath [9] approximated densities to support the validity of the equidistribution property of Corollary 3.

ExAMPLE 26. Consider the group $\langle 2\rangle \leqslant \mathbb{Q}^{\times}$. Focusing on the set of primes up to $10^{6}$, we find with SageMath the following approximated values for the density $\operatorname{dens}_{\mathbb{Q}}(2, a \bmod d)$ 
ANTONELLA PERUCCA-PIETRO SGOBBA

\begin{tabular}{|c||c|c|}
\hline$a \bmod d$ & $\operatorname{dens}_{\mathbb{Q}}(2, a \bmod d)$ & primes up to $10^{6}$ \\
\hline \hline $4 \bmod 16$ & $1 / 6 \approx 0.1667$ & 0.1676 \\
$12 \bmod 16$ & $1 / 6 \approx 0.1667$ & 0.1652 \\
\hline $3 \bmod 9$ & $1 / 8=0.125$ & 0.1236 \\
$6 \bmod 9$ & $1 / 8=0.125$ & 0.1266 \\
\hline $9 \bmod 27$ & $1 / 24 \approx 0.0417$ & 0.0422 \\
$18 \bmod 27$ & $1 / 24 \approx 0.0417$ & 0.0411 \\
\hline $3 \bmod 27$ & $1 / 24 \approx 0.0417$ & 0.0416 \\
$6 \bmod 27$ & $1 / 24 \approx 0.0417$ & 0.0421 \\
$15 \bmod 27$ & $1 / 24 \approx 0.0417$ & 0.0420 \\
$21 \bmod 27$ & $1 / 24 \approx 0.0417$ & 0.0405 \\
\hline
\end{tabular}

For instance, by Corollary 3, for $3 \mid a$ and $d=9$ or $d=27$ we have

$$
\operatorname{dens}_{\mathbb{Q}}(2, a \bmod d)=\frac{1}{\left[\mathbb{Q}\left(\zeta_{3}\right): \mathbb{Q}\right]} \cdot \operatorname{dens}_{\mathbb{Q}\left(\zeta_{3}\right)}(2, a \bmod d),
$$

and similarly for $4 \mid a$ and $d=16$. Thus we can compute these densities by following the same procedure as in the previous examples.

EXAMPLE 27. We consider the group $G=\langle 2,3\rangle \leqslant \mathbb{Q}^{\times}$and compute the densities $\operatorname{dens}_{\mathbb{Q}}(G, a \bmod d)$ using the methods of the previous examples. Again we study the set of primes up to $10^{6}$ and find with SageMath the following approximated values for the considered densities:

\begin{tabular}{|c||c|c|}
\hline$a \bmod d$ & $\operatorname{dens}_{\mathbb{Q}}(G, a \bmod d)$ & primes up to $10^{6}$ \\
\hline \hline $4 \bmod 16$ & $17 / 112 \approx 0.1518$ & 0.1522 \\
$12 \bmod 16$ & $17 / 112 \approx 0.1518$ & 0.1508 \\
\hline $3 \bmod 9$ & $2 / 13 \approx 0.1538$ & 0.1538 \\
$6 \bmod 9$ & $2 / 13 \approx 0.1538$ & 0.1540 \\
\hline $9 \bmod 27$ & $2 / 39 \approx 0.0513$ & 0.0513 \\
$18 \bmod 27$ & $2 / 39 \approx 0.0513$ & 0.0513 \\
\hline $3 \bmod 27$ & $2 / 39 \approx 0.0513$ & 0.0518 \\
$6 \bmod 27$ & $2 / 39 \approx 0.0513$ & 0.0512 \\
$15 \bmod 27$ & $2 / 39 \approx 0.0513$ & 0.0513 \\
$21 \bmod 27$ & $2 / 39 \approx 0.0513$ & 0.0507 \\
\hline
\end{tabular}

ExAmPLE 28. Let $K=\mathbb{Q}\left(\zeta_{3}\right)^{\times}$, and let $G$ be a finitely generated and torsion-free subgroup of $\mathbb{Q}\left(\zeta_{3}\right)^{\times}$. Consider the group $G^{\prime}=G \times\left\langle\zeta_{6}\right\rangle$. We study the density of primes $\mathfrak{p}$ of $K$ such that $\operatorname{ord}_{\mathfrak{p}}\left(G^{\prime}\right) \equiv a \bmod 10$, as considered in Section 4 . 


\section{KUMMER THEORY AND THE REDUCTIONS OF ALGEBRAIC NUMBERS II}

For $a=1,3,5,7,9$, we have $\operatorname{dens}_{K}^{\prime}(G, 6 ; a \bmod 10)=0$. For $a=4$ we have

$$
\begin{aligned}
\operatorname{dens}_{K}^{\prime}(G, 6 ; 4 \bmod 10)= & \operatorname{dens}_{K}^{\prime}(G, 2 ; 24 \bmod 30)+\operatorname{dens}_{K}^{\prime}(G, 2 ; 8 \bmod 10) \\
& -\operatorname{dens}_{K}^{\prime}(G, 2 ; 18 \bmod 30) \\
= & \operatorname{dens}_{K}\left(G^{2}, 12 \bmod 15\right)+\operatorname{dens}_{K}\left(G^{2}, 4 \bmod 5\right) \\
& -\operatorname{dens}_{K}\left(G^{2}, 9 \bmod 15\right),
\end{aligned}
$$

and also

$$
\begin{aligned}
\operatorname{dens}_{K}^{\prime}(G, 6 ; 4 \bmod 10)= & \operatorname{dens}_{K}^{\prime}\left(G^{2}, 3 ; 2 \bmod 5\right) \\
= & \operatorname{dens}_{K}\left(G^{2}, 12 \bmod 15\right)+\operatorname{dens}_{K}\left(G^{2}, 4 \bmod 5\right) \\
& -\operatorname{dens}_{K}\left(G^{2}, 9 \bmod 15\right)
\end{aligned}
$$

where the difference in the two calculations consists only in whether we consider the prime 2 or the prime 3 first for the method described in Section 4. For $a=2,6,8$ we can make a similar computation. Finally, for $a=0$ we have

$$
\operatorname{dens}_{K}^{\prime}(G, 6 ; 0 \bmod 10)=\operatorname{dens}_{K}(G, 0 \bmod 5),
$$

as 2 always divides $\operatorname{ord}_{\mathfrak{p}}\left(G^{\prime}\right)$, and

$$
\operatorname{ord}_{\mathfrak{p}}\left(G^{\prime}\right) \equiv 0 \bmod 5 \quad \text { if and only if } \quad \operatorname{ord}_{\mathfrak{p}}(G) \equiv 0 \bmod 5 .
$$

\section{REFERENCES}

[1] CHINEN, K.-MURATA, L.: On a Distribution Property of the Residual Order of a $(\bmod p) I V$. In: Papers from the 3rd China-Japan Seminar on Number Teory, Xi'an, China, February 12-16, 2004. (Zhang, Wenpeng, et al. eds), Number Theory. Tradition and Modernization. Developments in Math. Vol. 15, Springer, New York, NY, 2006.

[2] DEBRY, C.-PERUCCA, A.: Reductions of algebraic integers, J. Number Theory 167 (2016), 259-283.

[3] MOREE, P.: Artin's primitive root conjecture-a survey, Integers 12 (2012), no. 6, 1305-1416.

[4] MOREE, P.: On the distribution of the order and index of $g(\bmod p)$ over residue classes III, J. Number Theory 120 (2006), no. 1, 132-160.

[5] PERUCCA, A.: Multiplicative order and Frobenius symbol for the reductions of number fields, (J.S. Balakrishnan et al. eds.) In: Research Directions in Number Theory, Association for Women in Mathematics, Ser. 19 (2019), pp. 161-171.

[6] PERUCCA, A.: Prescribing valuations of the order of a point in the reductions of abelian varieties and tori. J. Number Theory 129 (2009), no. 2, 469-476.

[7] PERUCCA, A.: Reductions of algebraic integers II. (I. I. Bouw et al. eds.) In: Women in Numbers Europe II, Association for Women in Mathematics, Ser. 11 (2018), pp. 10-33. 


\section{ANTONELLA PERUCCA-PIETRO SGOBBA}

[8] PERUCCA, A.-SGOBBA, P.: Kummer theory for number fields and the reductions of algebraic numbers, Int. J. Number Theory, 15 (2019), no. 8, 1617-1633.

[9] SageMath-the Sage Mathematics Software System (Version 8.9). The Sage Developers, 2019, https://www.sagemath.org

[10] ZIEGLER, V.: On the distribution of the order of number field elements modulo prime ideals, Unif. Distrib. Theory 1 (2006), no. 1, 65-85.

Received July 7, 2019

Accepted March 23, 2020

\author{
Antonella Perucca \\ Pietro Sgobba \\ Department of Mathematics \\ Faculty of Science, Technology and \\ Medicine \\ University of Luxembourg \\ 6 av. de la Fonte \\ Esch-sur-Alzette 4364 \\ LUXEMBOURG \\ E-mail: antonella.perucca@uni.lu \\ pietro.sgobba@uni.lu
}

